\title{
Genetic architecture of maize kernel row number and whole genome prediction
}

\author{
Lei Liu ${ }^{1}$ Yanfang Du ${ }^{1}$ - Dongao Huo ${ }^{1} \cdot$ Man Wang $^{1} \cdot$ Xiaomeng Shen $^{1} \cdot$ Bing Yue $^{1} \cdot$ \\ ${\text { Fazhan } \text { Qiu }^{1} \text { - Yonglian Zheng }}^{1} \cdot$ Jianbing Yan $^{1} \cdot$ Zuxin Zhang $^{1}$
}

Received: 25 June 2014 / Accepted: 7 July 2015 / Published online: 19 July 2015

(C) The Author(s) 2015. This article is published with open access at Springerlink.com

\begin{abstract}
Key message Maize kernel row number might be dominated by a set of large additive or partially dominant loci and several small dominant loci and can be accurately predicted by fewer than 300 top $\mathrm{KRN}$-associated SNPs.

Abstract Kernel row number (KRN) is an important yield component in maize and directly affects grain yield. In this study, we combined linkage and association mapping to uncover the genetic architecture of maize KRN and to evaluate the phenotypic predictability using these detected loci. A genome-wide association study revealed 31 associated single nucleotide polymorphisms (SNPs) representing 17 genomic loci with an effect in at least one of five individual environments and the best linear unbiased prediction (BLUP) over all environments. Linkage mapping
\end{abstract}

Communicated by A. Charcosset.

Electronic supplementary material The online version of this article (doi:10.1007/s00122-015-2581-2) contains supplementary material, which is available to authorized users.

Zuxin Zhang

zuxinzhang@mail.hzau.edu.cn

Lei Liu

leil@webmail.hzau.edu.cn

Yanfang Du

yanfangdu@webmail.hzau.edu.cn

Dongao Huo

dongaohuo@gmail.com

Man Wang

15172538652@163.com

Xiaomeng Shen

891114sxm@webmail.hzau.edu.cn in three $\mathrm{F}_{2: 3}$ populations identified $33 \mathrm{KRN}$ quantitative trait loci (QTLs) representing 21 QTLs common to several population/environments. The majority of these common QTLs that displayed a large effect were additive or partially dominant. We found $70 \% \mathrm{KRN}$-associated genomic loci were mapped in KRN QTLs identified in this study, KRNassociated SNP hotspots detected in NAM population and/ or previous identified KRN QTL hotspots. Furthermore, the KRN of inbred lines and hybrids could be predicted by the additive effect of the SNPs, which was estimated using inbred lines as a training set. The prediction accuracy using the top KRN-associated tag SNPs was obviously higher than that of the randomly selected SNPs, and approximately 300 top KRN-associated tag SNPs were sufficient for predicting the KRN of the inbred lines and hybrids. The results suggest that the KRN-associated loci and QTLs that were detected in this study show great potential for improving the KRN with genomic selection in maize breeding.

Bing Yue

yuebing@webmail.hzau.edu.cn

Fazhan Qiu

qiufazhan@mail.hzau.edu.cn

Yonglian Zheng

zhyl@mail.hzau.edu.cn

Jianbing Yan

yjianbing@mail.hzau.edu.cn

National Key Laboratory of Crop Genetic Improvement, Huazhong Agricultural University, Wuhan 430070, China 


\section{Introduction}

Maize kernel row number (KRN) per ear is one of the most important yield components and is a breeding goal for the improvement of maize inbred lines. A better knowledge of the genetic architecture of KRN is required to establish breeding programs. However, current knowledge about the genetic control of the maize KRN was mainly obtained from genetic assays of inflorescence mutants. For example, thick tassel dwarf 1 (tdl) (Bommert et al. 2005) and fasciated ear 2 (fea2) (Taguchi-Shiobara et al. 2001; Bommert et al. 2013) are involved in meristem maintenance. The ramosa mutants can increase the indeterminacy of lateral organs, which transforms the determinate spikeletpair meristems into branches (Bortiri et al. 2006; Gallavotti et al. 2010; Satoh-Nagasawa et al. 2006; Sigmon and Vollbrecht 2010). Suppressor of sessile spikelets 1 (Sos1) controls meristem determinacy to produce single instead of paired spikelets in the inflorescence, thereby decreasing the KRN in the ear (Wu et al. 2009). The dominant Corngrass 1 (Cg1) mutant encodes two tandem zma-miR156 genes and leads to a small ear lacking an ordered kernel row and unbranched tassel (Chuck et al. 2007).

However, KRN in natural population displays a quantitative variation that is controlled by numerous QTLs. In the last 20 years, more than one hundred QTLs have been identified by linkage mapping (Veldboom and Lee 1994; Austin and Lee 1996; Ribaut et al. 1997; Yan et al. 2006; Lu et al. 2011a; Li et al. 2014). Despite the increasing accumulation of detected QTLs, the genetic architecture of the KRN has yet to be determined. In addition, the usefulness of these QTLs, which have mainly revealed the allelic variation between pairs of parents, is limited in maize breeding ( $\mathrm{Xu}$ and Crouch 2008). Recently, association mapping is being increasingly used in plants to uncover genetic effects of diverse alleles within a diverse population (Rafalski 2010). However, the population structure and the detection of rare variants are two major challenges for association mapping (Visscher 2008). A powerful approach that combines linkage analysis and association mapping has been developed to uncover the genetic architecture of complex quantitative traits in maize. For example, Brown et al. (2011) identified 36 QTLs and 261 significant SNPs for the KRN in a nested association mapping (NAM) population by a jointing linkage and genome-wide association study (GWAS).

Genome-wide genotyping also permits the improvement of the trait by genomic selection or whole-genome prediction (WGP), similar to that achieved in cattle breeding. This approach shows a great potential for crop improvement (Lorenzana and Bernardo 2009; Jannink et al. 2010). Previous studies have demonstrated that the KRN can be precisely predicted by genome-wide SNPs using an additive model in bi-parent populations (Riedelsheimer et al. 2013;
Guo et al. 2013). However, an additive model using hundreds of trait-associated SNPs cannot predict the KRN well in the NAM population (Brown et al. 2011). In this study, we employed an association panel and three designed $\mathrm{F}_{2: 3}$ populations to detect the loci involved in KRN variation, and used various marker sets (MSs) and training sets (TSs) to predict the KRN of maize inbred lines and hybrids. Our objectives were to dissect the genetic architecture of the KRN by GWAS and linkage mapping and to evaluate the predictability of WGP for the KRN of maize.

\section{Materials and methods}

\section{Genome-wide association study (GWAS)}

Panel 1, which is composed of 513 inbred lines (Table S1, Yang et al. 2011, the detailed information of these lines can be accessed in http://www.maizego.org/Resources.html), was evaluated for the KRN in five environments, Ya' an $\left(30^{\circ} \mathrm{N}, 103^{\circ} \mathrm{E}\right)$, Sanya $\left(18^{\circ} \mathrm{N}, 109^{\circ} \mathrm{E}\right)$ and Kunming $\left(25^{\circ} \mathrm{N}\right.$, $\left.102^{\circ} \mathrm{E}\right)$ in 2009 and Wuhan $\left(30^{\circ} \mathrm{N}, 114^{\circ} \mathrm{E}\right)$ and Kunming $\left(25^{\circ} \mathrm{N}, 102^{\circ} \mathrm{E}\right)$ in 2010 , under a randomized block design with two replicates. At harvesting stage, $8-10$ ears in each replicate were phenotyped for KRN. The average value of the two replicates was considered as phenotype of a given genotype, and average KRN in each environment was used to calculate the best linear unbiased prediction (BLUP) of KRN by the linear mixed models of the SAS software (SAS Institute Inc.). The average KRN in each environment and the BLUP results were then used for further research. The repeatability was calculated by $H^{2}=\delta_{\mathrm{g}}^{2} /\left(\delta_{\mathrm{g}}^{2}+\delta_{\mathrm{e}}^{2} / \mathrm{nr}\right)$ $\left(\delta_{g}^{2}\right.$ : genetic variance; $\delta_{e}^{2}$ : error; $n$ : number of environments, and $r$ : number of replicates).

The MaizeSNP50 Genotyping BeadChip was used for genotyping Panel 1 ( $\mathrm{Li}$ et al. 2012) and total of 48,962 SNPs with minor allelic frequencies $(\mathrm{MAF}) \geq 0.05$ were obtained. Both generalized (GLM) and mixed linear model (MLM) under a controlling population structure $(\mathrm{Q})$ and principal components analysis (PCA) (GLM, GLM + Q, GLM + PCA, MLM, MLM + Q and MLM + PCA) were applied to establish an association between the SNP and KRN using Tassel v. 3.0 (Yu et al. 2006; Bradbury et al. 2007; Zhang et al. 2010). The Meff method was performed using SNPSpD package to estimate the number of independent tests $\left(M_{\text {eff }}\right)$ for the 48962 SNPs of Panel 1 (Nyholt 2004; Li and Ji 2005). The stringent significance threshold was set to $0.05 / M_{\text {eff, }}$, which corresponds to a Bonferroni correction on Meff tests. In other studies of dent and flint maize panels conducted with the same SNP markers, this procedure led to approximately a tenth of the initial number of markers (Rincent et al. 2014). We therefore also considered $0.1 \mathrm{~N}$ as a reference value for comparison. 
Therefore, three less stringent thresholds, corresponding to $0.1 / M_{\text {eff }}, 0.05 / 0.1 \mathrm{~N}$ and $0.10 / 0.1 N$, were also set $(N$ total number of SNPs). All of kinship coefficient $(K)$, population structure $(Q)$ and principal components analysis (PCA) used in GWAS were estimated in a previous study (Yang et al. 2011) and top 10 axes of variations in PCA were employed in association analysis. The proportion of phenotype variance explained (PVE) by single SNP and all associated SNPs were estimated through a linear regression and corrected for population structure (Q matrix) as follow: $R^{2}=1-\mathrm{RSS} 1 / \mathrm{RSS} 0$ (Xu 2003). RSS0 is the residual sum of squares of a liner regression by population structure to phenotype, and RSS1 is the residual sum of squares of a liner regression by population structure and SNPs (single SNP or all associated SNPs) to phenotype (Xu 2003).

\section{Linkage mapping}

Three $\mathrm{F}_{2: 3}$ mapping populations were derived from NX531 $\times$ SIL8 $($ NS), TY6 $\times$ Mo17 $($ TM) and TY6 $\times$ W138 (TW) with 200, 188 and 284 families, respectively. NX531 and TY6 are two inbred lines with a high KRN, while SIL8, Mo17 and W138 have a low KRN. TY6, Mo17 and W138 were selected from Panel 1 based on the genotype at significant association sites. The number of favorable alleles at the significant association sites in TY6, Mo17 and W138 is listed in Table S3. Genomewide SSR markers, with 170 for NS, 199 for TM and 184 for TW, were used to genotype the three linkage mapping populations, respectively. The $\mathrm{F}_{2: 3}$ families of the NS were phenotyped at Xingtai $\left(38^{\circ} \mathrm{N}, 115^{\circ} \mathrm{E}\right)$ in 2011 ; both the TM and TW were phenotyped at Xingtai and Wuhan $\left(30^{\circ} \mathrm{N}\right.$, $114^{\circ} \mathrm{E}$ ) in 2012 using a randomized block design with three replicates. The broad-sense heritability was calculated by $\mathrm{H}_{\mathrm{b}}^{2}=\delta_{\mathrm{g}}^{2} /\left(\delta_{\mathrm{g}}^{2}+\delta_{\mathrm{ge}}^{2} / n+\delta_{\mathrm{e}}^{2} / n r\right)\left(\delta_{\mathrm{g}}^{2}\right.$ : genetic variance; $\delta_{\mathrm{ge}}^{2}$ : genotype $\times$ environment variance; $\delta_{\mathrm{e}}^{2}$ : error; $n$ : number of environments; $r$ : number of replicates). The linkage maps were constructed by MAPMAKER/EXP V3 (Lincoln et al. 1992), and then QTL mapping was conducted under additive and dominant model using the composite interval mapping (CIM) algorithm in the Windows QTL Cartographer 2.5 (Wang et al. 2012) with $5 \mathrm{cM}$ as window size and the threshold $\mathrm{LOD}=2.5$. A physical region which was repeatedly detected for KRN QTL in different populations was assumed as one common QTL. Previous identified KRN QTLs (Austin and Lee 1996; Veldboom and Lee 1996; Upadyayula et al. 2006; Yan et al. 2006; Liu et al. 2007; Ma et al. 2007; Tang et al. 2010; Tan et al. 2011; Lu et al. 2011a) were collected and projected to the B73 RefGen_v2 genome based on the physical location of flanking makers
(Table S6). The frequency of QTL detected repetitively was calculated within a $10 \mathrm{Mb}$ window size sliding every $5 \mathrm{Mb}$. A genomic region which was detected twice or more was defined as a KRN QTL hotspot.

\section{Genomic prediction for KRN}

To predict the KRN of the inbred lines and hybrids, we estimated the predictability by whole-genome prediction (WGP). First, the LD among 48,962 SNPs was estimated and was used to classify all of the SNPs to LD blocks based on the threshold $r^{2}>0.2$. The SNP that was most significantly associated with KRN in a given LD block was labeled as "tagSNP", and all of the tagSNPs were pooled as a marker bank to sample the marker sets (MSs). Then, we performed a WGP using the ridge-regression best linear unbiased prediction (RR-BLUP) using various MSs, training sets (TSs) and validation populations (VPs) (Piepho 2009; Endelman 2011). To compare effect of the MSs, we adopted two strategies to form the MSs and then to predict the KRN using 257 randomly selected lines (half of Panel 1) as TSs and both the remaining 256 lines in Panel 1 and the 54 single-cross hybrids as VPs (Fig. 1a). These 54 hybrids (Table S2) were produced by biparental crossing among 24 inbred lines, and were phenotyped in 2013 Xingtai $\left(38^{\circ} \mathrm{N}, 115^{\circ} \mathrm{E}\right)$. In Strategy 1, five to $24 \mathrm{~K}$ tagSNPs (referred to as the Top tagSNPs) were selected according to decreasing by significance $[-\log (p$ value $)]$. In Strategy 2, five to $24 \mathrm{~K}$ tagSNPs were randomly sampled from the marker bank by automatically incrementing 5 SNPs at the next sampling (Fig. 1a). The predictability of the same size of MS was determined by five repeated samplings. To evaluate the effect of the population structure on the WGP, we classified the lines in Panel 1 into two subpopulations: temperate lines and tropical/subtropical lines. Half of the lines of each subpopulation were randomly selected as TSs (temperate TS: Temp-TS; tropical/subtropical TS: TropTS) and the remaining lines as VPs (temperate VP: TempVP; tropical/subtropical VP: Trop-VP) (Fig. 1b). Moreover, we selected 300 Top tagSNPs as MS to evaluate the effect of the TS size. The size of the TSs was composed of randomly selected 50, 100, 150, 200, and 257 lines in Panel 1. The 54 hybrids and randomly selected 256 lines from the remainders in Panel 1 were sampled as VPs (Fig. 1c). Each of the above-mentioned prediction procedures was repeated 100 times. The KRN of the inbred lines that was evaluated in each five environments, the BLUP over environments and the KRN of 54 hybrids that were evaluated in 2013 at Xingtai were used as observed values. The correlation $(r)$ between the predicted and observed KRN was calculated to evaluate the predictability. 
Fig. 1 The pipelines of KRN prediction. a Prediction of the KRN in Panel 1 and the hybrids using top tagSNPs and randomly selected tagSNPs. b The effect of the population structure on genomic prediction. c The effect of the TS size on genomic prediction. $M S$ marker set, $T S$ training set, $V P$ validation population, Temp temperate lines, Trop tropical/subtropical lines
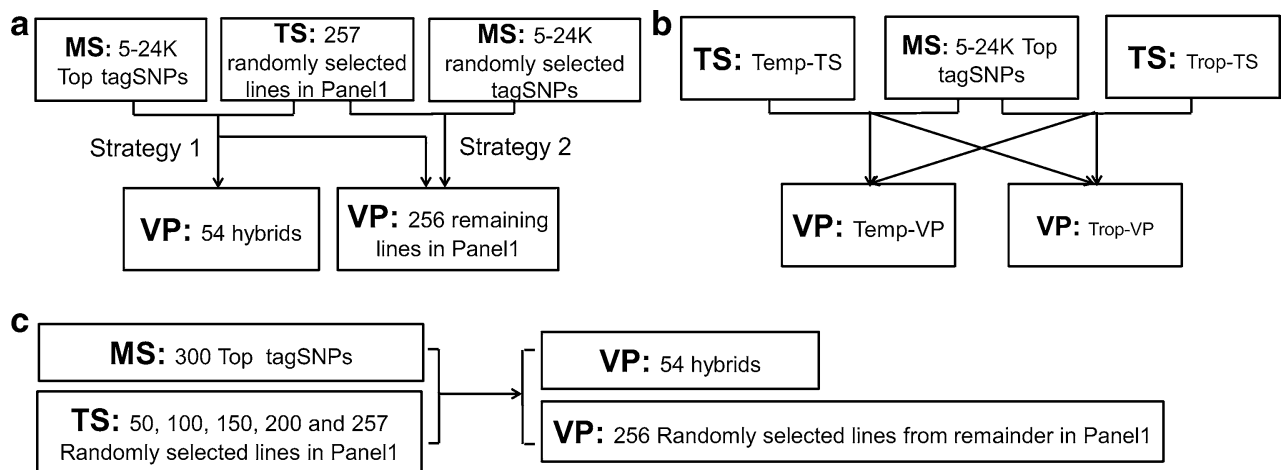

Table 1 The number of KRN significant associated SNPs under four thresholds

\begin{tabular}{|c|c|c|c|c|c|c|c|c|}
\hline Threshold $^{\mathrm{a}}$ & $-\log (p$ value $)$ & Yaan (2009) & Kunming (2009) & Sanya (2009) & Wuhan (2010) & Kunming (2010) & BLUP & Total \\
\hline $0.1 / 0.1 \mathrm{~N}$ & 4.69 & 5 & 8 & 18 & 3 & 1 & 7 & 31 \\
\hline $0.05 / 0.1 \mathrm{~N}$ & 4.99 & 4 & 7 & 13 & 3 & 0 & 4 & 24 \\
\hline $0.1 / M_{\mathrm{eff}}$ & 5.69 & 0 & 2 & 6 & 0 & 0 & 0 & 7 \\
\hline $0.05 / M_{\mathrm{eff}}$ & 5.99 & 0 & 2 & 5 & 0 & 0 & 0 & 7 \\
\hline
\end{tabular}

$M_{\text {eff }}=48498.7009$ (the number of independent test); $N=48962$ (the total SNPs number)

a The Bonferroni correction thresholds were corrected by the Meff method (Nyholt 2004; Li and Ji 2005)

\section{Results}

\section{Genome-wide association study}

The KRN phenotypes of Panel 1 were evaluated under five environments and showed a normal distribution ranging from 9.3 to 19.7 rows per ear, with an average of $13.3 \pm 1.6$ (Table S3). Importantly, the observed KRN values indicated high repeatability $(93.87 \%)$ (Table S3) with significant correlations $(r=0.69-0.85)$ among the five environments (Table S4). As in the study of Yang et al. (2011), Panel 1 can be divided into four subpopulations: Tropical-subtropical (TST), Stiff Stalk (SS), Nonstiff stalk (NSS), and Admix. The KRN of inbred lines in the SS (mid-value 14.0, ranging from 11.1 to 15.7), Admix (mid-value 13.6, ranging from 12.6 to 14.9 ) and NSS (mid-value 13.3, ranging from 12.3 to 14.2 ) was higher than that in TST (mid-value 12.8, ranging from 11.8 to 13.6 ) (Figure $\mathrm{S} 1$ ), indicating the influence of population structure for KRN. About 8.04-26.77\% of KRN variances under different environments could be explained by population structure, indicating that population structure might affect the association analysis of KRN (Table S3).

To determine the appropriate model for GWAS, six models (see "Materials and methods") were evaluated in Panel 1. Compared with GLM model, the three MLM models showed a stronger control for Type I error (Figure $\mathrm{S} 2$ ). In previous studies, the MLM $+\mathrm{Q}$ model was successfully applied for GWAS of quantitative traits in Panel 1 (Li et al. 2013; Wen et al. 2014). Therefore, this model was also chosen for GWAS in this study (Figure S2). The number of independent tests $\left(M_{\text {eff }}\right)$ for the 48962 SNPs of Panel 1 was 48498.7, similar to total SNPs number. This result was different from the finding of Rincent et al., who found a larger decrease in $M_{\text {eff }}$ compared to total SNPs number (2014). Genetic diversity in an association population likely influences the number of independent tests. Thus, the estimation of independent tests for various genetic materials deserves further investigation. The $0.05 / M_{\text {eff }}$ level of Bonferroni corrected $-\log (p$ value) was 5.99 (Table 1$)$, and the less stringent $-\log (p$ value) thresholds, corresponding to $0.1 / M_{\text {eff }}, 0.05 / 0.1 N$ and $0.10 / 0.1 N$, were $5.69,4.99$ and 4.69 , respectively (Table 1). Under the four thresholds 7, 7, 24 and $31 \mathrm{KRN}$ associated SNPs were identified, respectively (Table 1). In consideration of possible over correction of the MLM + Q model and narrow KRN variation in Panel 1, the threshold $-\log (p$ value $) 4.69$ was used to identify a larger number of KRN-associated SNPs, thereby the 31 KRN-associated SNPs were considered for further investigation. The proportion of phenotype variance explained (PVE) by individual SNP ranged from 2.45 to $10 \%$, and 24 SNPs had a PVE $>5 \%$ (Table S5). In Panel 1, frequency of the allele with positive effect at these 31 SNP sites ranged from 0.09 to 0.79 and was less than 0.3 for $68 \%$ of the sites. Interestingly, frequency of the allele with positive effect at the KRN-associated SNPs was 
Table 2 Summary of the KRN-associated genomic loci by the GWAS in Panel 1

\begin{tabular}{|c|c|c|c|c|c|c|c|}
\hline Genomic loci $^{\mathrm{a}}$ & SNP Number ${ }^{b}$ & $\mathrm{Chr}^{\mathrm{c}}$ & Env $^{\mathrm{d}}$ & Significant level ${ }^{e}$ & $\operatorname{PVE}(\%)^{\mathrm{f}}$ & Co-localized QTLs ${ }^{\mathrm{g}}$ & $\begin{array}{l}\text { Co-localization with } \\
\text { previous loci }^{\text {h }}\end{array}$ \\
\hline$q K R N 1$ & 1 & 1 & KM2010 & 4.69 & 4.75 & $q K R N 1-2$ & $* \#$ \\
\hline$q K R N 2$ & 1 & 2 & BLUP & 4.69 & 2.45 & & $\#$ \\
\hline$q K R N 3 a$ & 1 & 3 & YA2009 & 4.69 & 4.50 & & $\&$ \\
\hline$q K R N 3 b$ & 2 & 3 & YA2009 & 4.99 & 8.16 & $q K R N 3-2$ & $* \#$ \\
\hline$q K R N 4 a$ & 1 & 4 & WH2010 & 4.99 & 2.48 & & $\&$ \\
\hline$q K R N 4 b$ & 4 & 4 & KM\&SY2009 & 4.99 & 9.30 & $q K R N 4-1$ & $\&$ \\
\hline$q K R N 4 c$ & 1 & 4 & KM2009 & 4.99 & 5.72 & & $\&$ \\
\hline$q K R N 4 d$ & 1 & 4 & WH2010 & 4.99 & 3.89 & & $\&$ \\
\hline$q K R N 4 e$ & 10 & 4 & SY2009\&BLUP & 5.99 & 9.38 & $q K R N 4-4$ & $* \#$ \\
\hline$q K R N 4 f$ & 1 & 4 & YA2009 & 4.99 & 4.50 & & $* \#$ \\
\hline$q K R N 5$ & 1 & 5 & SY2009 & 4.69 & 9.66 & & $* \#$ \\
\hline$q K R N 6 a$ & 1 & 6 & SY2009 & 4.69 & 10.00 & & $\#$ \\
\hline$q K R N 6 b$ & 1 & 6 & SY2009 & 4.99 & 6.60 & $q K R N 6-2$ & $* \#$ \\
\hline$q K R N 6 c$ & 1 & 6 & YA\&KM2009 & 5.99 & 9.28 & $q K R N 6-2$ & $* \#$ \\
\hline$q K R N 9 a$ & 1 & 9 & BLUP & 4.99 & 7.00 & & $* \#$ \\
\hline$q K R N 9 b$ & 2 & 9 & KM2009 & 5.99 & 9.93 & & $* \#$ \\
\hline$q K R N 10$ & 1 & 10 & WH2010 & 4.99 & 4.79 & & \\
\hline
\end{tabular}

a Genomic loci were referred by LD SNPs or neighbor SNPs

b Associated SNPs number at each genomic locus

c Chromosome of the KRN-associated genomic loci

d The environments of associated loci detected, YA Yaan; KM Kunming; SY Sanya; WH Wuhan

e The significant associated thresholds $-\log (p$ value) of the SNPs in each KRN-associated genomic loci could reach

$\mathrm{f}$ The maximum phenotype variance explanation (PVE) of SNPs in each KRN-associated loci across five environments and BLUP

g Co-localized QTLs detected in NS, TM and TW

h Co-localize with previous identified QTLs, “*” represents co-localization with KRN-associated SNPs hotspots in NAM population (Brown et al. 2011); “\#” represents co-localization with previous identified KRN QTL hotspots; \& represents co-localization with previous identified QTLs (See "Materials and methods")

negatively correlated with phenotypic variation explained by these sites $(r=-0.63, p<0.0001)$. Considering the criterion of LD with $r^{2}>0.2$, the 31 SNPs belonged to 17 LD blocks which represented $17 \mathrm{KRN}$-associated genomic loci (Table 2, Fig. 2) including 4 loci covered by more than one KRN-associated SNP and 13 loci supported by single KRN-associated SNP (Table 2, Table S5). A total $58.8 \%(10 / 17)$ of $\mathrm{KRN}$-associated genomic loci had a PVE $>5 \%$ (Table 2). Furthermore, multiple regressions indicated that $51.10-62.05 \%$ of the phenotypic variation was explained by these 17 genomic loci in the five environments and the BLUP over all environments, respectively (Table S5).

\section{Linkage analysis}

Three $\mathrm{F}_{2: 3}$ populations (NS, TM, and TW) were developed by crossing high KRN lines with low KRN lines to map the KRN QTLs. In each of the $\mathrm{F}_{2: 3}$ mapping populations, ANOVA analysis also revealed significant genetic variation in the KRN among the $F_{2: 3}$ families. The KRN ranged from 10.2 to 17.4 in NS, 10.7 to 18.1 in TM and 10.0 to 19.2 in TW (Table S3, Figure S1). Additionally, the KRN in the TM and TW populations at Wuhan were highly correlated with that at Xingtai (Table S3), and exhibited high broad-sense heritability with $88.39 \%$ in TM and $78.03 \%$ in TW (Table S3). The SSR markers (170 for NS, 199 for TM and 184 for TW) were used for the linkage and QTL mapping. A total of $1836.7 \mathrm{cM}-, 2277.7 \mathrm{cM}$ - and $2258.9 \mathrm{cM}-$ length genetic maps were constructed for the NS, TM and TW, with an average distance among the markers of 10.8, 11.4 and 12.3 in NS, TM and TW, respectively. A total of 33 QTLs were detected in the three linkage populations, 7 in the NS, 13 in the TM and 13 in the TW (Table 3). Single QTL explained 2.3-28.4\% of phenotype variance (Table 3), and $94 \%(31 / 33)$ of QTLs had a PVE $>5 \%$ (Table 3 ). About $63.6 \%(21 / 33)$ of the QTLs were 


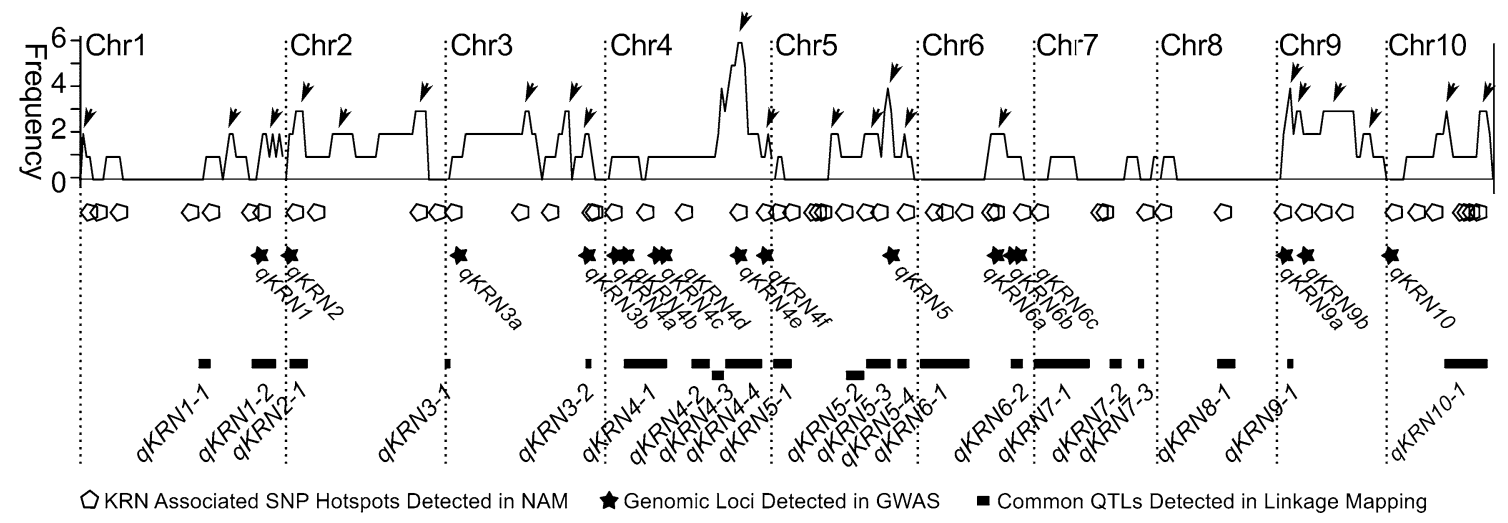

Fig. 2 The distribution of genetic loci for KRN detected in this study and previous studies. The $X$-axis represents chromosomes of maize, and the $Y$-axis represents the frequency of KRN QTL repetitively detected on a certain genomic region by previous studies (Table S6). The black arrowhead points out the previous identified KRN

additive or partially dominant, while $36.4 \%(12 / 33)$ of the QTLs were completely dominant (Table 3 ). All dominant loci also displayed largely additive effects. Furthermore, the 33 QTLs were clustered into 21 common QTLs based on the genomic locations of the flanking markers. Of the 21 common QTLs, 12 QTLs had a PVE $>5 \%$ and 6 QTLs had a PVE $>10 \%$ (Table 3). Five QTLs (qKRN4-3, qKRN4-4, qKRN5-1, qKRN5-4 and qKRN62) were detected across two environments, and four QTLs ( $q$ KRN4-4, qKRN5-1, qKRN5-4 and qKRN10-1) were detected in two or more populations (Table 3 ).

\section{Co-localization between QTL and associated genomic loci for KRN}

The flanking markers of common QTLs detected in this study were projected to the B73 RefGen_v2 to anchor the QTL physical location. We found that $61.3 \%(19 / 31)$ of the KRN-associated SNPs or $35 \%(6 / 17)$ of the KRNassociated genomic loci ( $q K R N 1, q K R N 3 b, q K R N 4 b$, $q K R N 4 e, q K R N 6 b$ and $q K R N 6 c$ ) co-localized with 5 common QTLs (Table 2; Fig. 2). Furthermore, 75 KRN QTLs and $261 \mathrm{KRN}$-associated SNPs that were identified in previous studies were collected and were then projected to the B73 RefGen_v2. This projection resulted in $22 \mathrm{KRN}$ QTL hotspots and $50 \mathrm{KRN}$-associated SNPs hotspots (Fig. 2). A total of $65 \%(11 / 17) \mathrm{KRN}$-associated genome loci fell into the KRN QTL hotspots, among these, five co-localized with QTL regions identified in previous studies (Fig. 2; Table 2). Nine KRN-associated genomic loci co-localized with KRN-associated SNP hotspots which were detected in NAM population (Fig. 2; Table 2). Resultantly, $70 \%$ (12/17) KRN-associated genomic loci were cross-validated
QTL hotspot. Pentagons KRN-associated SNPs hotspots detected in the NAM population (Brown et al. 2011). The black stars and black boxes represent $\mathrm{KRN}$-associated loci detected in GWAS and common QTLs detected in linkage mapping in this study, respectively

by linkage mapping QTLs in this study, as well as by KRN QTL hotspots and KRN-associated SNPs in earlier investigations (Fig. 2; Table 2).

\section{Genomic prediction for KRN}

According to the $\mathrm{LD}$ value, 48,962 SNPs with MAF $>5 \%$ were grouped into $24,521 \mathrm{LD}$ blocks which were represented by 24,521 tagSNPs to generate MSs (marker sets). Using the MS comprising of 17 SNPs, which represented $31 \mathrm{KRN}$-associated SNPs in the GWAS (Table 4), the prediction accuracies of the KRN were $0.48-0.60$ for the inbred lines and 0.64-0.69 for the hybrids (Fig. 3a, b). When the MSs increased from 5 to 300 top tagSNPs, the prediction accuracies of the inbred lines and hybrids increased sharply and reached to the highest level $(r=0.78)$ (Fig. 3a, b; Table 4), while the prediction accuracies of the inbred lines decreased when more than $2 \mathrm{~K}$ top tagSNPs were used. In addition, the prediction accuracies also increased quickly when 5-200 randomly selected tagSNPs were used, increased slowly when the tagSNPs increased from 200 to $1 \mathrm{~K}(r<0.35)$, and rapidly increased again when the randomly selected tagSNPs increased from $1 \mathrm{~K}$ to $24 \mathrm{~K}$ (Fig. 3a; Table 4). These results suggest that the prediction accuracy of the randomly selected tagSNPs was lower than that of the top tagSNPs, and that a small subset of markers (approximately 300 top tagSNPs) might be required for the KRN prediction of the inbred lines; additional markers could not improve the predictability. For the KRN prediction of the hybrids, a higher prediction accuracy $(r=0.76)$ was observed when $\sim 40$ top tagSNPs were used and was maintained at a high level along with increasing the top tagSNPs (Fig. 3c; Table 4), indicating 
Table 3 KRN QTLs those were detected in three $\mathrm{F}_{2: 3}$ families

\begin{tabular}{|c|c|c|c|c|c|c|c|c|c|c|c|c|}
\hline QTL & Env & Pop & $\mathrm{Chr}$ & $\begin{array}{l}\text { Flanking } \\
\text { markers }\end{array}$ & $\begin{array}{l}\text { Genetic } \\
\text { interval cM }\end{array}$ & $\begin{array}{l}\text { Physical } \\
\text { interval Mb }\end{array}$ & LOD & A & $\mathrm{D}$ & $\operatorname{PVE}(\%)$ & $\mathrm{d} / \mathrm{a}^{\mathrm{a}}$ & $\begin{array}{l}\text { Gene } \\
\text { action }\end{array}$ \\
\hline$q K R N 1-1$ & $2012 \mathrm{XT}$ & $\mathrm{TM}$ & 1 & umc2396-bnlg1025 & 36 & 24.4 & 7.83 & 0.73 & -0.29 & 9.67 & 0.39 & $\mathrm{pd}$ \\
\hline$q K R N 1-2$ & $2012 \mathrm{XT}$ & TW & 1 & umc1184-M1.108 & 12.7 & 37.6 & 2.77 & -0.4 & 0.39 & 2.49 & 0.97 & $\mathrm{~d}$ \\
\hline$q K R N 2-1$ & $2010 \mathrm{XT}$ & NS & 2 & bnlg1537-prp2 & 27.9 & 24.3 & 6.07 & 0.56 & 0.06 & 8.82 & 0.11 & $\mathrm{a}$ \\
\hline$q K R N 3-1$ & $2010 \mathrm{XT}$ & NS & 3 & phi37411-bnlg1325 & 30.7 & 4 & 6.68 & 0.55 & 0.04 & 8.44 & 0.07 & $\mathrm{a}$ \\
\hline$q K R N 3-2$ & 2012 XT & $\mathrm{TW}$ & 3 & umc2152-umc 2048 & 22.4 & 9.9 & 4.87 & 0.41 & -0.03 & 4.21 & 0.07 & a \\
\hline$q K R N 4-1$ & 2012 XT & TW & 4 & umc1117-umc1791 & 12.7 & 71.1 & 2.77 & 0.2 & 0.24 & 2.33 & 1.21 & $\mathrm{~d}$ \\
\hline$q K R N 4-2$ & $2012 \mathrm{XT}$ & $\mathrm{TW}$ & 4 & umc1346-umc1137 & 13.6 & 26.8 & 2.95 & 0.31 & 0.26 & 3.04 & 0.84 & $\mathrm{~d}$ \\
\hline$q K R N 4-3$ & $2012 \mathrm{WH}$ & $\mathrm{TM}$ & 4 & umc1329-umc1194 & 27.5 & 14.4 & 5.98 & 0.49 & 0.1 & 12.67 & 0.21 & a \\
\hline qKRN4-3 & $2012 \mathrm{XT}$ & $\mathrm{TM}$ & 4 & bnlg1137-umc1086 & 58.1 & 22.8 & 12.63 & 0.71 & 0.11 & 18.54 & 0.16 & a \\
\hline$q K R N 4-4$ & $2012 \mathrm{XT}$ & TW & 4 & umc1194-umc2188 & 40.4 & 30.6 & 26.65 & 1.24 & -0.09 & 26.05 & 0.08 & a \\
\hline$q K R N 4-4$ & $2012 \mathrm{WH}$ & TW & 4 & umc1194-umc2188 & 54.3 & 30.6 & 11.8 & 0.91 & -0.01 & 18.34 & 0.02 & a \\
\hline$q K R N 4-4$ & $2010 \mathrm{XT}$ & NS & 4 & bnlg2162-umc1284 & 63.6 & 49.7 & 13.82 & 0.78 & 0.07 & 20.71 & 0.09 & a \\
\hline$q K R N 5-1$ & $2012 \mathrm{WH}$ & $\mathrm{TM}$ & 5 & umc1097-umc 2578 & 18 & 17.9 & 3.92 & 0.17 & 0.27 & 7.31 & 1.55 & $\mathrm{~d}$ \\
\hline$q K R N 5-1$ & $2010 \mathrm{XT}$ & NS & 5 & umc1365-umc1464 & 66.7 & 8.9 & 14.49 & 1.2 & -0.17 & 28.43 & 0.14 & $\mathrm{a}$ \\
\hline$q K R N 5-1$ & $2012 \mathrm{XT}$ & TW & 5 & umc2036-umc 2578 & 39.1 & 13.2 & 8.51 & 0.36 & 0.34 & 7.71 & 0.93 & $\mathrm{~d}$ \\
\hline$q K R N 5-1$ & $2012 \mathrm{XT}$ & $\mathrm{TM}$ & 5 & umc1587-umc1056 & 19.5 & 21.5 & 4.25 & 0.41 & -0.04 & 5.51 & 0.11 & $\mathrm{a}$ \\
\hline$q K R N 5-1$ & $2012 \mathrm{WH}$ & TW & 5 & umc1894-umc1056 & 29.2 & 19.8 & 6.35 & 0.45 & 0.05 & 7.4 & 0.1 & $\mathrm{a}$ \\
\hline$q K R N 5-2$ & $2012 \mathrm{WH}$ & TW & 5 & umc1056-umc1389 & 26.1 & 36.7 & 5.68 & 0.38 & 0.2 & 7.33 & 0.54 & $\mathrm{pd}$ \\
\hline qKRN5-3 & 2012 XT & $\mathrm{TM}$ & 5 & umc1171-bnlg1306 & 38.3 & 38.7 & 8.32 & 0.78 & -0.26 & 11.78 & 0.34 & $\mathrm{pd}$ \\
\hline$q K R N 5-4$ & $2012 \mathrm{WH}$ & TW & 5 & umc1941-umc1072 & 14.1 & 19.2 & 3.06 & 0.46 & -0.05 & 5.48 & 0.11 & $\mathrm{a}$ \\
\hline$q K R N 5-4$ & $2012 \mathrm{XT}$ & TW & 5 & umc1941-umc1072 & 25.2 & 19.2 & 5.49 & 0.57 & -0.14 & 6.71 & 0.25 & $\mathrm{a}$ \\
\hline$q K R N 5-4$ & $2012 \mathrm{WH}$ & $\mathrm{TM}$ & 5 & bnlg1306-umc1072 & 17.2 & 2.6 & 3.74 & 0.6 & -0.33 & 7.79 & 0.55 & $\mathrm{pd}$ \\
\hline$q K R N 5-4$ & 2012 XT & $\mathrm{TM}$ & 5 & bnlg1306-umc1072 & 11.9 & 2.6 & 2.58 & 0.66 & -0.23 & 4.11 & 0.34 & $\mathrm{pd}$ \\
\hline$q K R N 6-1$ & 2012 XT & TW & 6 & umc1143-umc1595 & 45.6 & 91.9 & 9.91 & 0.53 & 0.18 & 8.44 & 0.34 & $\mathrm{pd}$ \\
\hline$q K R N 6-2$ & 2012 XT & $\mathrm{TM}$ & 6 & umc1020-umc1296 & 34 & 15.4 & 7.4 & 0.45 & 0.26 & 9.74 & 0.58 & $\mathrm{pd}$ \\
\hline$q K R N 6-2$ & $2012 \mathrm{WH}$ & $\mathrm{TM}$ & 6 & umc1859-umc1248 & 20.8 & 14.8 & 4.53 & 0.24 & 0.4 & 9.96 & 1.66 & $\mathrm{~d}$ \\
\hline$q K R N 7-1$ & $2012 \mathrm{XT}$ & $\mathrm{TM}$ & 7 & mmc0171-umc1929 & 13.9 & 103.9 & 3.01 & -0.29 & 0.5 & 3.34 & 1.71 & $\mathrm{~d}$ \\
\hline$q K R N 7-2$ & $2010 \mathrm{XT}$ & NS & 7 & umc1125-umc1407 & 12.7 & 3.4 & 2.76 & 0.26 & 0.27 & 4.46 & 1.03 & $\mathrm{~d}$ \\
\hline$q K R N 7-3$ & $2012 \mathrm{XT}$ & $\mathrm{TM}$ & 7 & umc1983-bnlg1022 & 12.5 & 23.5 & 2.71 & -0.27 & 0.54 & 3.66 & 1.99 & $\mathrm{~d}$ \\
\hline$q K R N 8-1$ & $2010 \mathrm{XT}$ & NS & 8 & bnlg2181-umc2199 & 15.1 & 26.7 & 3.29 & 0.39 & -0.57 & 5.39 & 1.47 & $\mathrm{~d}$ \\
\hline$q K R N 9-1$ & $2012 \mathrm{XT}$ & $\mathrm{TM}$ & 9 & umc1170-umc1037 & 12.6 & 6.1 & 2.74 & -0.01 & 0.48 & 4.38 & 48 & $\mathrm{~d}$ \\
\hline$q K R N 10-1$ & 2010 XT & NS & 10 & bnlg1526-umc1506 & 20.3 & 15.4 & 4.4 & 0.05 & 0.42 & 4.96 & 7.91 & $\mathrm{~d}$ \\
\hline$q K R N 10-1$ & $2012 \mathrm{WH}$ & TW & 10 & umc1995-umc1640 & 19.5 & 56 & 4.24 & 0.65 & 0.33 & 15.51 & 0.51 & $\mathrm{pd}$ \\
\hline
\end{tabular}

ENV Environment, $X T$ Xingtai, $W H$ Wuhan, Pop population, $C h r$ chromosome, A Additive effect, $D$ Dominant effect, $P V E$ phenotype variance that was explained, $p d$ partial dominant effect, $d$ dominant effect

a The degree of dominance, $\mathrm{d} / \mathrm{a}$ (dominant effect/additive effect) (Stuber et al. 1987)

that the KRN of the hybrids can be predicted by the additive effect of the SNPs which were estimated using inbred lines as a training set.

To evaluate the effect of both the TS and VP in the WGP, we compared the predictability of various combinations between the TS and VP. First, when the TSs and VPs were divided by population structure (Temp-TS, TempVP, Trop-TS and Trop-VP), the prediction accuracies increased sharply with the increasing number of the top tagSNPs from 5 to 300 and decreased with the increasing number of the top tagSNPs from $1 \mathrm{~K}$ to $24 \mathrm{~K}$. For the temp-VP or trop-VP, the prediction accuracies were almost the same when the temp-TS and trop-TS were used, respectively. Moreover, no matter whether the tempTS or trop-TS was used, the prediction accuracies were always higher for the temp-VP than for the trop-VP. Second, with the increasing TS size, the accuracies increased slowly in the prediction of the inbred lines but increased quickly in the prediction of the hybrids (Figs. 1c, 3d), demonstrating that the TS size affects the predictability of whole-genome prediction, consistent with the report of Riedelsheimer et al. (2013). 
Table 4 The prediction accuracies (\%) using the top tagSNPs and randomly selected tagSNPs for the inbred lines and hybrids

\begin{tabular}{|c|c|c|c|c|c|c|c|}
\hline SNP number & Prediction & 2009 (Yaan) & 2009 (Kunming) & 2009 (Sanya) & 2010 (Kunming) & 2010 (Wuhan) & BLUP \\
\hline \multirow[t]{3}{*}{17} & Strategy 1 for Panel 1 & 53 & 60 & 59 & 48 & 49 & 57 \\
\hline & Strategy 1 for Hybrids & 64 & 65 & 69 & 59 & 64 & 68 \\
\hline & Strategy 2 for Panel 1 & 9 & 10 & 15 & 9 & 14 & 13 \\
\hline \multirow[t]{3}{*}{300} & Strategy 1 for Panel 1 & 73 & 71 & 78 & 66 & 70 & 77 \\
\hline & Strategy 1 for Hybrids & 71 & 69 & 67 & 75 & 69 & 71 \\
\hline & Strategy 2 for Panel 1 & 27 & 26 & 33 & 22 & 31 & 31 \\
\hline \multirow[t]{3}{*}{$1 \mathrm{~K}$} & Strategy 1 for Panel 1 & 73 & 72 & 78 & 68 & 73 & 78 \\
\hline & Strategy 1 for Hybrids & 73 & 71 & 72 & 71 & 74 & 73 \\
\hline & Strategy 2 for Panel 1 & 31 & 29 & 37 & 27 & 35 & 35 \\
\hline \multirow[t]{3}{*}{$10 \mathrm{~K}$} & Strategy 1 for Panel 1 & 68 & 66 & 72 & 61 & 68 & 73 \\
\hline & Strategy 1 for Hybrids & 73 & 69 & 73 & 77 & 75 & 77 \\
\hline & Strategy 2 for Panel 1 & 46 & 44 & 50 & 40 & 47 & 50 \\
\hline \multirow[t]{3}{*}{$20 \mathrm{~K}$} & Strategy 1 for Panel 1 & 56 & 54 & 61 & 49 & 58 & 61 \\
\hline & Strategy 1 for Hybrids & 75 & 70 & 72 & 76 & 74 & 77 \\
\hline & Strategy 2 for Panel 1 & 45 & 43 & 50 & 41 & 47 & 50 \\
\hline \multirow[t]{3}{*}{$24 \mathrm{~K}$} & Strategy 1 for Panel 1 & 56 & 53 & 61 & 49 & 57 & 60 \\
\hline & Strategy 1 for Hybrids & 73 & 70 & 72 & 76 & 74 & 78 \\
\hline & Strategy 2 for Panel 1 & 55 & 53 & 61 & 49 & 57 & 61 \\
\hline
\end{tabular}

Strategy 1 for Panel 1: using the top tagSNPs to predict the inbred lines in Panel 1; Strategy 1 for Hybrids: using the top tagSNPs to predict the hybrids; Strategy 2 for Panel 1: using the randomly selected tagSNPs to predict the inbred lines in Panel 1

\section{Discussion}

\section{Genetic architecture of the maize kernel row number}

Since the first application of molecular marker technology in QTL mapping in the 1980s, numerous QTLs for agriculturally important traits have been identified in diverse populations. The QTLs for KRN have been widely assayed, and hundreds of QTLs have been identified (Veldboom and Lee 1994; Austin and Lee 1996; Ribaut et al. 1997; Yan et al. 2006; Lu et al. 2011a; Li et al. 2014). In general, investigations using segregation populations that were derived from biparental cross, such as RILs, BCn, and DH, frequently identified only few major QTLs ( 10\% PVE) and several minor QTLs due to limited allelic effect differences between the two parents. Evidence from the GWAS in the maize NAM population indicates that the flowering time (Buckler et al. 2009), resistance to southern leaf blight (Poland et al. 2011) and leaf architecture (Tian et al. 2011) are dominated by small additive QTLs with few genetic or environmental interactions. In this study, KRN of maize exhibited a high repeatability and a relatively narrow variation range, and was influenced by population structure in Panel 1. By GWAS, we found about 51.10-62.05\% KRN variance in Panel 1 was dominated by 17 genomic loci, of which 10 loci had a PVE $>5 \%$ (Table 2, Table S5). In Panel 1, frequency of positive allele of the $\mathrm{KRN}$-associated SNPs ranged from 0.09 to 0.79 , was negatively correlated with its PVE, and most of SNP sites (68\%) had a low frequency of positive allele $(<0.3)$ (Table S5), suggesting that those SNPs with low frequency seem to have a large genetic effect for KRN. A similar result was also observed by Brown et al. (2011), who determined that those loci for inflorescence traits have larger effects than do the flowering- and leaf trait-associated loci, and these large effect alleles had low frequency. This result indicated that favorable alleles of the KRN-associated loci were held by a few inbred lines in Panel 1. As described by Yang et al. (2011), Panel 1 is composed of a set of elite inbred lines, including parental lines of high vigor hybrids, inbred lines utilizing in maize breeding program, and improving lines from the germplasm enhancement of maize project (GEM). A low frequency of positive allele at the KRN-associated SNPs in Panel 1 implicated that favorable KRN alleles have not yet been fully integrated into elite inbred lines. Therefore, those KRN-associated SNPs and QTLs detected in this study give us target loci for improving KRN of elite inbred lines of maize.

The consistency between the association loci from the GWAS and QTLs from linkage mapping provides a crossvalidation of the mapping results from the two approaches and also indicates the important loci for the KRN. In this study, three linkage mapping populations were used to validate the GWAS results. We found that $\sim 70 \%$ of the $\mathrm{KRN}$-associated genomic loci detected in this study were cross-validated by KRN QTLs identified in the three $\mathrm{F}_{2: 3}$ 

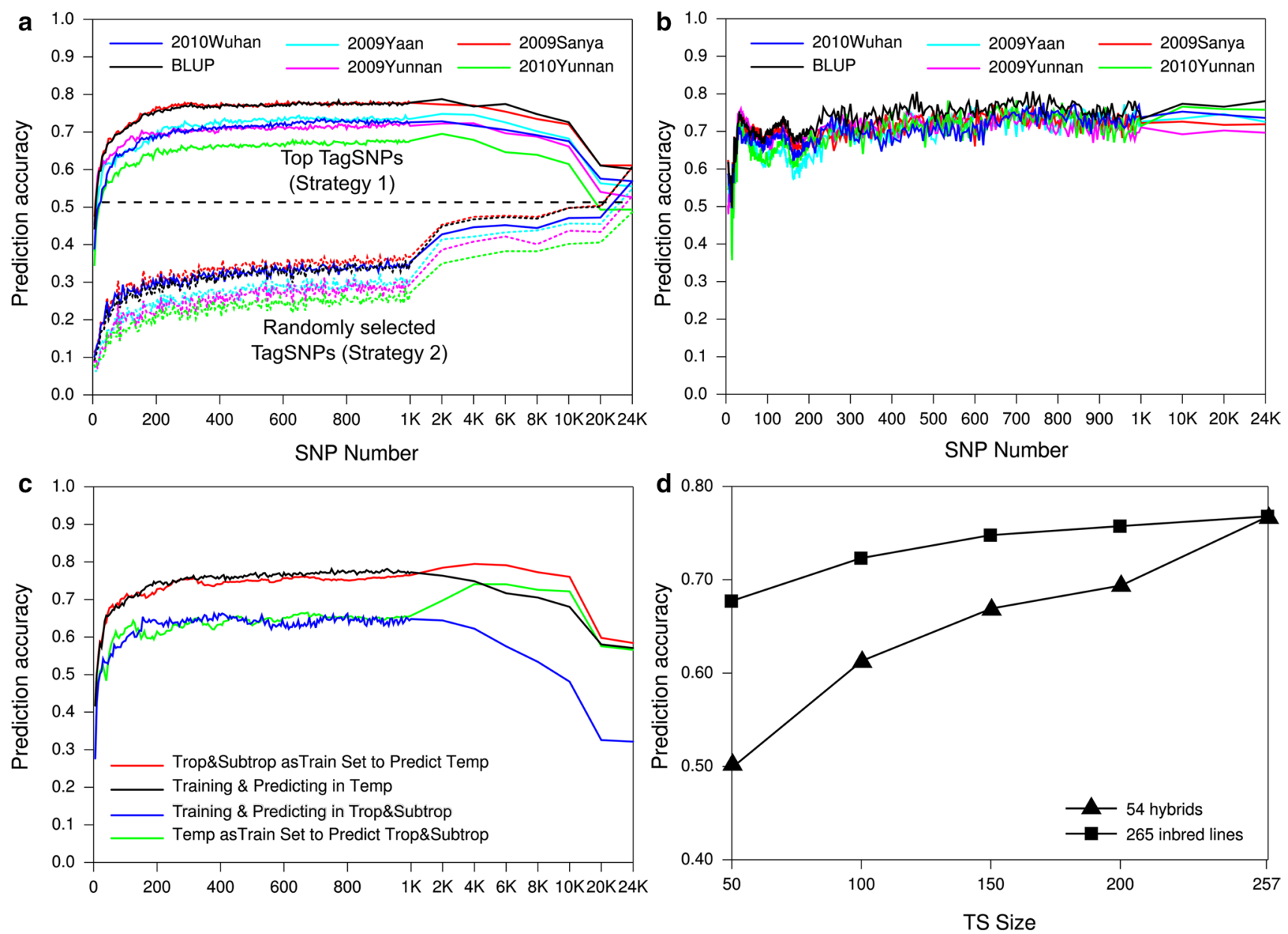

Fig. 3 Predictability using tagSNPs for the kernel row number. a Predictability of the top tagSNPs and randomly selected tagSNPs in the inbred lines. Continuous lines the prediction accuracies using 5-24 K top tagSNPs (strategy 1); Dotted lines the prediction accuracies using 5-24 K randomly selected tagSNPs (strategy 2). b Predic-

families, KRN QTLs hotspots observed in previous studies and KRN-associated SNP hotspots detected in NAM population (Fig. 2; Table 2). A total of $48 \%$ of common QTLs were proved to be consistent with previously identified QTLs hotspots (Fig. 2). Integrating the KRN QTLs that were detected in the previous and present studies by linkage and GWAS, we found more than 40 loci, comprising some of the large additive loci ( $>3 \%)$ and many small additive loci $(<3 \%)$, which dominate the natural variation in the KRN in maize.

On the basis of the degree of dominance (d/a), QTLs that were detected in the linkage populations were divided into three types: additive, partial dominant, and dominant (Stuber et al. 1987). Of the 33 QTLs that were detected in this study, 13 QTLs (39.4\%) were additive, 8 QTLs (24.2\%) were partially dominant and 12 QTLs $(36.4 \%)$ were dominant or overdominant. A total of 6 of the 13 additive tion accuracies using 5-24 K top tagSNPs for 54 hybrids. c Prediction accuracies of 5-24 K top tagSNPs in different subpopulations using different training sets and validation populations. $\mathbf{d}$ Predictability of different sizes of training sets using 300 top tagSNPs in inbred lines and hybrids

QTLs $(46.2 \%)$ had a PVE $>10 \%$, ranging from 12.67 to $26.05 \%$, while 4 of the 12 partial dominant QTLs (33.3\%) had a PVE close to or greater than $10 \%$, ranging from 9.67 to $15.51 \%$. Conversely, only one dominant QTL showed a PVE close to $10 \%$ (Table 3), clearly indicating that QTL with additive and partial dominant effect play a major role in the genetic architecture of the KRN.

\section{Whole-genome prediction of the kernel row number}

One of the objectives of the identification of genetic loci is to use those loci to guide the improvement of important traits in maize hybrid breeding. Brown et al. (2011) suggested that an additive model showed a low predictive ability for KRN in NAM. However, in this study, we found that the prediction accuracies of the KRN in the maize inbred lines and hybrids were higher $(r=0.66-0.78)$ than in the 
NAM population (Brown et al. 2011) and were similar to the predictability in the DH and RIL lines (Riedelsheimer et al. 2013; Guo et al. 2013). Particularly, a high predictability could be achieved by selecting a small subset of top tagSNPs (100-300 top tagSNPs). A similar conclusion was also observed in the NAM population for ear trait prediction (Brown et al. 2011), demonstrating the importance of trait-associated loci (SNPs) for WGP. Earlier studies have suggested that the proportion of genetic variance that is explained by the SNPs is important for the accuracy of the WGP (Campos et al. 2010; Riedelsheimer et al. 2013). For 17 of the 300 top tagSNPs that were detected in the GWAS, the prediction accuracies were $0.48-0.60$ for the inbred lines and $0.64-0.69$ for the hybrids. The predictability could be improved by $18 \%$ by the other 283 top tagSNPs that failed to be detected in the GWAS. These results also support the hypothesis that the maize KRN in natural population is controlled by some of large additive loci and many of minor additive loci. In addition, the additive model that was used in this study showed a high predictability for the KRN of inbred lines and hybrids, indicating that the KRN might be mainly controlled by additive loci.

Compared with the randomly selected tagSNPs, the top tagSNPs could effectively improve the prediction accuracy (Fig. 2a). Interestingly, when the number of top tagSNPs was increased to more than $1 \mathrm{~K}$, the predictability for the KRN of inbred lines in Panel 1 decreased to $R^{2}<0.65$, which could be obtained by selecting just $<50$ top tagSNPs (Fig. 3a). This result demonstrates that a small subset of the trait-associated markers is required for the KRN prediction of inbred lines and randomly high-density SNPs as a marker set cannot improve the predictability in the WGP. A possible interpretation is that numerous neutral SNPs, which are not associated with a trait, cannot genetically improve the proportion of the variance that is explained by SNPs. Therefore, a small subset of top tagSNPs, which can effectively save cost and time, should be recommended for the WGP of the KRN in maize molecular breeding.

Both size of TS and relatedness between TS and VP, which strongly influence the accuracy of SNP effects, are important for predictability in genomic selection (Clark et al. 2012; Riedelsheimer et al. 2013). For natural population, population structure is also a key factor for dissecting genetic architecture (Rafalski 2010). Inbred lines in Panel 1 could be divided by the population structure and adaptiveness into temperate and tropical and subtropical lines. Although temperate lines and tropical and subtropical lines show high genetic divergence (Lanza et al. 1997; Lu et al. 2011b), the prediction accuracies for the temp-VP or trop-VP were almost the same no matter whether tempTS or trop-TS was used (Fig. 3c). However, the prediction accuracies for trop-VP were lower than that for temp-VP
(Fig. 3d). Two main factors might result in the decline of predictive ability for trop-VP: (1) the rapid LD decay in tropical and subtropical lines attenuated the relationship between LD tagSNPs and causal variants, led to more false positive SNPs detected which, in turn, strongly reduced the predictive ability (Lu et al. 2011a; Campos et al. 2010). (2) The greater variability among tropical and subtropical lines relative to temperate lines (Lanza et al. 1997) might affect the accuracy of effects of causal variants. In addition, we found that the prediction accuracies were highly positively correlated with the TS size in this study (Fig. 3d), indicating a large sample of the TS is required for the WGP.

In conclusion, by GWAS in Panel 1, we identified $31 \mathrm{KRN}$-associated SNPs which represented $17 \mathrm{KRN}$ associated genomic loci. In the $17 \mathrm{KRN}$-associated loci, 16 loci were validated by 21 common QTLs which were identified from three linkage populations (TM, TW and NS) and 22 KRN QTL hotspots identified in previous studies. Although none of variations in these loci were confirmed to be the causal variations for KRN, those Top tagSNPs from GWAS were successfully employed to predict KRN of inbred lines and hybrids using RR-BLUP. Moreover, we found a high predictability can be achieved through selecting hundreds of the Top tagSNPs in inbred lines and hybrids, suggesting the Top tagSNPs should be potential target loci for KRN improvement with genomic selection in maize breeding.

Author contribution statement LL, JY, and ZZ carried out the GWAS of the KRN in Panel 1 and Panel 2 and drafted the manuscript. LL, YD, DH, MW, XC and XS participated in the linkage mapping. LL, ZZ and YZ participated in the design of the study and the interpretation of the results and wrote and edited the manuscript.

Acknowledgments We are grateful to Dr Xiaohong Yang (China Agricultural University, China) for data analysis and Dr. Hongwu Wang (Chinese Academy of Agricultural Science, China), Dr. Sibin Gao (Sichuan Agricultural University, China), Dr. Zhangyong Liu and Dr. Hewei Du (Yangtze University, China) for assistance in the phenotype evaluation. This work was supported by the National basic research program of China (2014CB138203) and the National Natural Science Foundation of China (91335110).

\section{Compliance with ethical standards}

Conflict of interest The authors declare that they have no conflict of interest.

Open Access This article is distributed under the terms of the Creative Commons Attribution 4.0 International License (http://creativecommons.org/licenses/by/4.0/), which permits unrestricted use, distribution, and reproduction in any medium, provided you give appropriate credit to the original author(s) and the source, provide a link to the Creative Commons license, and indicate if changes were made. 


\section{References}

Austin DF, Lee M (1996) Comparative mapping in $\mathrm{F}_{2: 3}$ and $\mathrm{F}_{6: 7}$ generations of quantitative trait loci for grain yield and yield components in maize. Theor Appl Genet 92:817-826

Bommert P, Lunde C, Nardmann J, Vollbrecht E, Running M, Jackson D, Hake S, Werr W (2005) thick tassel dwarfl encodes a putative maize ortholog of the Arabidopsis CLAVATA1 leucine-rich repeat receptor-like kinase. Development 132:1235-1245

Bommert P, Nagasawa NS, Jackson D (2013) Quantitative variation in maize kernel row number is controlled by the FASCIATED EAR2 locus. Nat Genet 45(3):334-337

Bortiri E, Chuck G, Vollbrecht E, Rocheford T, Martienssen R, Hake $S$ (2006) ramosa2 encodes a lateral organ boundary domain protein that determines the fate of stem cells in branch meristems of maize. Plant Cell 18:574-585

Bradbury PJ, Zhang Z, Kroon DE, Casstevens TM, Ramdoss Y, Buckler ES (2007) TASSEL: software for association mapping of complex traits in diverse samples. Bioinformatics 23:2633-2635

Brown PJ, Upadyayula N, Mahone GS, Tian F, Bradbury PJ, Myles S, Holland JB, Flint-Garcia S, McMullen MD, Buckler ES, Rocheford TR (2011) Distinct genetic architectures for male and female inflorescence traits of maize. PLoS Genet 7:e1002383

Buckler ES, Holland JB, Bradbury PJ, Acharya CB, Brown PJ, Browne C, Ersoz E, Flint-Garcia S, Garcia A, Glaubitz JC, Goodman MM, Harjes C, Guill K, Kroon DE, Larsson S, Lepak NK, Li H, Mitchell SE, Pressoir G, Peiffer JA, Rosas MO, Rocheford TR, Romay MC, Romero S, Salvo S, Sanchez Villeda H, da Silva HS, Sun Q, Tian F, Upadyayula N, Ware D, Yates H, Yu J, Zhang Z, Kresovich S, McMullen MD (2009) The genetic architecture of maize flowering time. Science 325:714-718

Campos G, Gianola D, Allison DB (2010) Predicting genetic predisposition in humans: the promise of whole-genome markers. Nature Rev Genet 11:880-886

Chuck G, Cigana M, Saeteurn K, Hake S (2007) The heterochronic maize mutant Corngrass 1 results from overexpression of a tandem microRNA. Nat Genet 39:544-549

Clark SA, Hickey JM, Daetwyler HD, van der Werf JHJ (2012) The importance of information on relatives for the prediction of genomic breeding values and the implications for the makeup of reference data sets in livestock breeding schemes. Genet Sel Evol 44:4. doi:10.1186/1297-9686-44-4

Endelman JB (2011) Ridge regression and other kernels for genomic selection with R package rrBLUP. Plant Genome 4:250-255

Gallavotti A, Long JA, Stanfield S, Yang X, Jackson D, Vollbrecht E, Schmidt RJ (2010) The control of axillary meristem fate in the maize ramosa pathway. Stem Cells Dev 2856:2849-2856

Guo T, Li H, Yan J, Tang J, Li J, Zhang Z, Zhang L, Wang J (2013) Performance prediction of $\mathrm{F}_{1}$ hybrids between recombinant inbred lines derived from two elite maize inbred lines. Theor Appl Genet 126(1):189-201

Jannink JC, Lorenz AJ, Iwata H (2010) Genomic selection in plant breeding: from theory to practice. Brief Funct Genomic 9:166-177

Lanza LLB, deSouza CL, Ottoboni LMM, Vieira MLC, deSouza AP (1997) Genetic distance of inbred lines and prediction of maize single-cross performance using RAPD markers. Theor Appl Genet 94:1023-1030

Li J, Ji L (2005) Adjusting multiple testing in multilocus analyses using the eigenvalues of a correlation matrix. Heredity 95:221-227

Li Q, Yang X, Xu S, Cai Y, Zhang D, Han Y, Li L, Zhang Z, Gao S, Li J, Yan J (2012) Genome-wide association studies identified three independent polymorphisms associated with $\alpha$-tocopherol content in maize kernels. PLoS One 7:e36807
Li H, Peng Z, Yang X et al (2013) Genome-wide association study dissects the genetic architecture of oil biosynthesis in maize kernels. Nat Genet 45:43-50. doi:10.1038/ng.2484

Li F, Jia H, Liu L, Zhang C, Liu Z, Zhang Z (2014) Quantitative trait loci mapping for kernel row number using chromosome segment substitution lines in maize. Genet Mol Res 13(1):1707-1716

Lincoln S, Daly M, Lander E (1992) Constructing genetics maps with MAPMAKER/EXP 3.0. Whitehead institute technical report. Whitehead Institute, Cambridge

Liu ZH, Tang JH, Wei XY, Wang CL, Tian GW, Hu YM, Chen WC (2007) QTL mapping of ear traits under low and high nitrogen conditions in maize. Sci Agric Sin 40(11):2409-2417

Lorenzana RE, Bernardo R (2009) Accuracy of genotypic value predictions for marker-based selection in biparental plant populations. Theor Appl Genet 120:151-161

Lu M, Xie C, Li X, Hao Z, Li M, Weng J, Zhang D, Bai L, Zhang S (2011a) Mapping of quantitative trait loci for kernel row number in maize across seven environments. Mol Breed 28:143-152

Lu Y, Shah T, Hao Z et al (2011b) Comparative SNP and haplotype analysis reveals a higher genetic diversity and rapider LD decay in tropical than temperate germplasm in maize. PLoS One. doi:10.1371/journal.pone.0024861

Ma XQ, Tang JH, Teng WT, Yan JB, Meng YJ et al (2007) Epistatic interaction is an important genetic basis of grain yield and its components in maize. Mol Breed 20:41-51

Nyholt DR (2004) A simple correction for multiple testing for SNPs in linkage disequilibrium with each other. Am J Hum Genet 74(4):765-769

Piepho HP (2009) Ridge regression and extensions for genomewide selection in maize. Crop Sci 49:1165-1176

Poland JA, Bradbury PJ, Buckler ES, Nelson RJ (2011) Genome-wide nested association mapping of quantitative resistance to northern leaf blight in maize. Proc Natl Acad Sci USA 108:6893-6898

Rafalski JA (2010) Association genetics in crop improvement. Curr Opin Plant Biol 13:174-180

Ribaut JM, Jiang C, Gonzalez-de-Leon D, Edmeades GO, Hoisington DA (1997) Identification of quantitative trait loci under drought conditions in tropical maize. 2. Yield components and markerassisted selection strategies. Theor Appl Genet 94:887-896

Riedelsheimer C, Endelman JB, Stange M, Sorrells ME, Jannink JL, Melchinger AE (2013) Genomic predictability of interconnected biparental maize populations. Genetics 194:493-503

Rincent R, Nicolas S, Bouchet S, Moreau L, Charcosset A et al (2014) Dent and flint maize diversity panels reveal important genetic potential for increasing biomass production. Theor Appl Genet 127:2313-2331

Satoh-Nagasawa N, Nagasawa N, Malcomber S, Sakai H, Jackson D (2006) A trehalose metabolic enzyme controls inflorescence architecture in maize. Nature 441:227-230

Sigmon B, Vollbrecht E (2010) Evidence of selection at the ramosa1 locus during maize domestication. Mol Ecol 19:1296-1311

Stuber CW, Edwards MD, Wendel JF (1987) Molecular markerfacilitated investigation of quantitative trait loci in maize. II. Factors influencing yields and its component traits. Crop Sci 27:639-644

Taguchi-Shiobara F, Yuan Z, Hake S, Jackson D (2001) The fasciated ear2 gene encodes a leucine-rich repeat receptor-like protein that regulates shoot meristem proliferation in maize. Genes Dev 15:2755-2766

Tan WW, Wang Y, Li YX, Liu C, Liu ZZ, Peng B, Wang D, Zhang Y, Sun BC, Shi YS, Song YC, Wang TY, Li Y (2011) QTL analysis of ear traits in maize across multiple environments. Sci Agric Sin 44(2):233-244

Tang JH, Yan JB, Ma XQ, Teng WT, Wu WR, Dai JR, Dhillon BS, Melchinger AE, Li JS (2010) Dissection of the genetic basis of 
heterosis in an elite maize hybrid by QTL mapping in an immortalized F2 population. Theor Appl Genet 120:333-340

Tian F, Bradbury PJ, Brown PJ, Hung H, Sun Q, Flint-Garcia S, Rocheford TR, McMullen MD, Holland JB, Buckler ES (2011) Genome-wide association study of leaf architecture in the maize nested association mapping population. Nat Genet 43:159-162

Veldboom LR, Lee M (1994) Molecular-marker-facilitated studies of morphological traits in maize. II. Determination of QTLs for gain yield and yield components. Theor Appl Genet 89(4):451-458

Veldboom LR, Lee M (1996) Genetic mapping of quantitative trait loci in maize in stress and nonstress environments.1. Grain yield and yield components. Crop Sci 36(5):1310-1319

Visscher PM (2008) Sizing up human height variation. Nat Genet 40:489-490

Wang S, Basten CJ, Zeng ZB (2012) Windows QTL Cartographer 2.5. Department of Statistics, North Carolina State University, Raleigh, NC (http://statgen.ncsu.edu/qtlcart/ WQTLCart.htm)

Wen W, Li D, Li X et al (2014) Metabolome-based genome-wide association study of maize kernel leads to novel biochemical insights. Nat Commun 5:3438. doi:10.1038/ncomms4438

Wu X, Skirpan A, McSteen P (2009) Suppressor of sessile spikelets1 functions in the ramosa pathway controlling meristem determinacy in maize. Plant Physiol 149:205-219
Xu R (2003) Measuring explained variation in linear mixed effects models. Stat Med 22:3527-3541

Xu YB, Crouch J (2008) Marker-assisted selection in plant breeding: from publications to practice. Crop Sci 48:391-407

Yan J, Tang H, Huang Y, Zheng Y, Li J (2006) QTL mapping and epistatic analysis for yield and yield components using molecular markers with an elite maize hybrid. Euphytica 149:121-131

Yang X, Gao S, Xu S, Zhang Z, Prasanna B, Li L, Li J, Yan J (2011) Characterization of a global germplasm collection and its potential utilization for analysis of complex quantitative traits in maize. Mol Breed 28:511-526

Yu J, Pressoir G, Briggs WH, Vroh Bi I, Yamasaki M, Doebley JF, McMullen MD, Gaut BS, Nielsen DM, Holland JB, Kresovich S, Buckler ES (2006) A unified mixed-model method for association mapping that accounts for multiple levels of relatedness. Nat Genet 38:203-208

Zhang Z, Ersoz E, Lai CQ, Todhunter RJ, Tiwari HK, Gore MA, Bradbury PJ, Yu J, Arnett DK, Ordovas JM, Buckler ES (2010) Mixed linear model approach adapted for genome-wide association studies. Nat Genet 42:355-360 